\title{
Introverted subspaces of the duals of measure algebras
}

\author{
H. JAVANSHIRI AND R. NASR-ISFAHANI
}

\begin{abstract}
Let $\mathcal{G}$ be a locally compact group. In continuation of our studies on the first and second duals of measure algebras by the use of the theory of generalised functions, here we study the $\mathrm{C}^{*}$-subalgebra $G L_{0}(\mathcal{G})$ of $G L(\mathcal{G})$ as an introverted subspace of $M(\mathcal{G})^{*}$. In the case where $\mathcal{G}$ is non-compact we show that any topological left invariant mean on $G L(\mathcal{G})$ lies in $G L_{0}(\mathcal{G})^{\perp}$. We then endow $G L_{0}(\mathcal{G})^{*}$ with an Arens-type product which contains $M(\mathcal{G})$ as a closed subalgebra and $M_{a}(\mathcal{G})$ as a closed ideal which is a solid set with respect to absolute continuity in $G L_{0}(\mathcal{G})^{*}$. Among other things, we prove that $\mathcal{G}$ is compact if and only if $G L_{0}(\mathcal{G})^{*}$ has a non-zero left (weakly) completely continuous element.
\end{abstract}

Mathematics Subject Classification (2010). 43A10, 43A15, 43A20, $47 \mathrm{~B} 07$.

Key words. Measure algebra, generalised functions vanishing at infinity, introverted subspace, topological invariant mean, completely continuous element.

\section{Introduction}

All over this paper $\mathcal{G}$ is a locally compact group with left Haar measure $\lambda$ and identity element $e$, and the notations $C_{c}(\mathcal{G})$ and $C_{0}(\mathcal{G})$ refer to the space of all functions with compact support and the space of all functions vanishing at infinity, respectively. Moreover, the letter $M(\mathcal{G})$ means that the measure algebra of $\mathcal{G}$ consisting of all complex regular Borel measures on $\mathcal{G}$ with the total variation norm and the convolution product " $*$ " defined by the formula

$$
\langle\mu * \nu, g\rangle=\int_{\mathcal{G}} \int_{\mathcal{G}} g(x y) d \mu(x) d \nu(y)
$$

for all $\mu, \nu \in M(\mathcal{G})$ and $g \in C_{0}(\mathcal{G})$. It is folklore that $M(\mathcal{G})$ is the first dual space of $C_{0}(\mathcal{G})$ for the pairing

$$
\langle\mu, g\rangle:=\int_{\mathcal{G}} g(x) d \mu(x) \quad\left(\mu \in M(\mathcal{G}), g \in C_{0}(\mathcal{G})\right) .
$$

In the last thirty years, research on the second duals of Banach algebras has mostly centred around the Banach algebras related to locally compact groups, and has been dealt with by Lau et al. in the works $[3,4,7,11]$. In particular, [7] is the first important work devoted to the study of the second duals of measure algebras. Among other things, the authors of [7] have conjectured that the Banach algebra $M(\mathcal{G})$ is strongly Arens irregular, and its second dual, $M(\mathcal{G})^{* *}$, determines $\mathcal{G}$ in the category of all locally compact groups. Later on, the second duals of the measure algebras has been studied in a series of papers. In particular, [4] is the second important work devoted to the study of $M(\mathcal{G})^{* *}$, where most of the known results about these 
Banach algebras up to the year 2012 can be found in it. Recall from [4] that $M(\mathcal{G})^{*}$, the first dual space of $M(\mathcal{G})$, as the second dual of the $\mathrm{C}^{*}$-algebra $C_{0}(\mathcal{G})$ is a commutative unital $\mathrm{C}^{*}$ algebra, and therefore if $\widetilde{\mathcal{G}}$ denotes the hyper-Stonean envelope of $\mathcal{G}$, then we can recognizing $M(\mathcal{G})^{*}$ as $C(\widetilde{\mathcal{G}})$, the space of all bounded complex-valued continuous functions on $\widetilde{\mathcal{G}}$. It follows that $M(\mathcal{G})^{* *} \cong M(\widetilde{\mathcal{G}})$, where $\cong$ denotes the isometric algebra isomorphism and many authors, up to the year 2012, have used a type of this identification as a tool for the study of $M(\mathcal{G})^{* *}$, see [4] and the references therein for more details.

Recently in the works $[6,10]$, we studied the first and second duals of measure algebras by the use of the theory of generalised functions which have been introduced and investigated by Šreidr [14] and Wong $[15,16]$. In those papers we observed that $G L_{0}(\mathcal{G})$, the space of all generalised functions that vanishes at infinity, plays a crucial role in our investigation. Motivated by this, here we study the $\mathrm{C}^{*}$-algebra $G L_{0}(\mathcal{G})$ as an introverted subspace of $M(\mathcal{G})^{*}$. In particular, in the case where $\mathcal{G}$ is non-compact we show that any topological left invariant mean on $G L(\mathcal{G})$ lies in $G L_{0}(\mathcal{G})^{\perp}$ which demonstrates that the weak*-closed subspace $G L_{0}(\mathcal{G})^{\perp}$ of $M(\mathcal{G})^{*}$ is far from devoid of interest. We then endow $G L_{0}(\mathcal{G})^{*}$ with an Arens-type product which contains $M(\mathcal{G})$ and $M_{a}(\mathcal{G})$ as a closed subalgebra and a closed ideal, respectively. Among other things, we prove that the existence of a non-zero left (weakly) completely continuous element in $G L_{0}(\mathcal{G})^{*}$ is equivalent to the compactness of $\mathcal{G}$.

\section{Generalised functions: an overview}

In this section, we give a brief overview of generalised functions in the sense of Wong [15]. Nevertheless, we shall require some facts about the theory of $\mathrm{C}^{*}$-algebra. For background on this theory, we use [13] as a reference and adopt that book's notation. Moreover, our notation and terminology are standard and, concerning Banach algebras related to locally compact groups, they are in general those of the book [9] of Hewitt and Ross. The reader will remark that this section is mostly taken from the papers by Wong $[15,16]$; this is because of, we actually need this section for the convenience of citation and a better exposition.

For any complex regular Borel measure $\mu$ on $\mathcal{G}$, let $L^{\infty}(|\mu|)$ denote the Banach space of all essentially bounded $|\mu|$-measurable complex functions $f_{\mu}$ on $\mathcal{G}$ with the essential supremum norm

$$
\left\|f_{\mu}\right\|_{\mu, \infty}=\inf \left\{\alpha \geq 0:\left|f_{\mu}\right| \leq \alpha,|\mu|-\text { a.e. }\right\} .
$$

Consider the product linear space $\prod\left\{L^{\infty}(|\mu|): \mu \in M(\mathcal{G})\right\}$. An element $f=\left(f_{\mu}\right)_{\mu \in M(\mathcal{G})}$ in this product is called a generalised function if $f_{\mu}=f_{\nu}|\mu|-$ a.e. for any $\mu, \nu \in M(\mathcal{G})$ with $\mu \ll \nu$, where $\mu \ll \nu$ means that $|\mu|$ is absolutely continuous with respect to $|\nu|$. We note that this condition implies that for given generalised functions $f=\left(f_{\mu}\right)_{\mu \in M(\mathcal{G})}$

$$
\sup \left\{\left\|f_{\mu}\right\|_{\mu, \infty}: \mu \in M(\mathcal{G})\right\}<\infty
$$

otherwise, there is a sequence $\left(\mu_{n}\right)$ in $M(\mathcal{G})$ for which $\left\|f_{\mu_{n}}\right\|_{\mu_{n}, \infty} \geq n$ for all $n \in \mathbb{N}$. Set $\mu=\sum_{n=1}^{\infty} 2^{-n}\left\|\mu_{n}\right\|^{-1}\left|\mu_{n}\right|$. Then $\mu_{n} \ll \mu$, and hence $\left\|f_{\mu}\right\|_{\mu, \infty} \geq\left\|f_{\mu_{n}}\right\|_{\mu_{n}, \infty} \geq n$ for all $n \in \mathbb{N}$ which is a contradiction. 
Now, following Wong [15] we use the notation $G L(\mathcal{G})$ to denote the commutative unital $\mathrm{C}^{*}$ algebra of all generalised functions endowed with the coordinatewise operations, the involution $f \mapsto f^{*}$, where $f^{*}:=\left(\overline{f_{\mu}}\right)_{\mu \in M(\mathcal{G})}$, and norm

$$
\|f\|_{\infty}:=\sup \left\{\left\|f_{\mu}\right\|_{\mu, \infty}: \mu \in M(\mathcal{G})\right\},
$$

where $f=\left(f_{\mu}\right)_{\mu \in M(\mathcal{G})}$ is in $G L(\mathcal{G})$. The identity element of $G L(\mathcal{G})$ is of course the generalised function $1:=\left(1_{\mu}\right)_{\mu \in M(\mathcal{G})}$, where $1_{\mu}$ is the identity element of $L^{\infty}(|\mu|)$. Moreover, we write $f=\left(f_{\mu}\right)_{\mu \in M(\mathcal{G})} \geq 0$ to mean that the generalised function $f$ is positive in the $\mathrm{C}^{*}$-algebra sense and denote by $G L(\mathcal{G})^{+}$the set of all positive elements of $G L(\mathcal{G})$.

Remark 2.1 It is not hard to check that a generalised function $f=\left(f_{\mu}\right)_{\mu \in M(\mathcal{G})}$ is positive in the $\mathrm{C}^{*}$-algebra sense if and only if $f_{\mu}(x) \geq 0$ for all $x \in \mathcal{G}$ and all $\mu \in M(\mathcal{G})$; see [13, Page. 45] and [15, Page. 85] for more information.

As a main result, Wong [15] has shown that for each $f=\left(f_{\mu}\right)_{\mu \in M(\mathcal{G})}$ in $G L(\mathcal{G})$, the equation

$$
\langle\Psi(f), \zeta\rangle:=\int_{\mathcal{G}} f_{\zeta}(x) d \zeta(x) \quad(\zeta \in M(\mathcal{G}))
$$

defines a linear functional $\Psi(f)$ on $M(\mathcal{G})$. In particular, the map $f \mapsto \Psi(f)$ is an isometric linear mapping from $G L(\mathcal{G})$ onto $M(\mathcal{G})^{*}$; see [15, Theorem 2.1 and Theorem 2.2] and [14] for the same result in the special case where $\mathcal{G}$ is a certain locally compact abelian group. In particular, any $L \in M(\mathcal{G})^{*}$ can be considered as a generalised function $\Psi^{-1}(L)$, and we do not distinguish between a generalised function $f$ and its unique corresponding linear functional $\Psi(f)$. In particular, this duality allows us to consider $G L(\mathcal{G})$ as a Banach $M(\mathcal{G})$-bimodule. In details, if $\zeta$ and $f=\left(f_{\mu}\right)_{\mu \in M(\mathcal{G})}$ are arbitrary elements of $M(\mathcal{G})$ and $G L(\mathcal{G})$, respectively. Then, one can considered the linear functionals $f \zeta$ and $\zeta f$ on $M(\mathcal{G})$ defined by

$$
\langle f \zeta, \mu\rangle=\langle f, \zeta * \mu\rangle, \quad\langle\zeta f, \mu\rangle=\langle f, \mu * \zeta\rangle \quad(\mu \in M(\mathcal{G})) .
$$

In order to find the generalised functions corresponding to these linear functionals, following Wong [15] and [16, Page 610], we define $\zeta \circ f \in \prod\left\{L^{\infty}(|\mu|): \mu \in M(\mathcal{G})\right\}$ as

$$
(\zeta \circ f)_{\mu}=l_{\zeta} f_{\zeta * \mu} \quad(\mu \in M(\mathcal{G})),
$$

where

$$
l_{\zeta} f_{\zeta * \mu}(y)=\int_{\mathcal{G}} f_{\zeta * \mu}(x y) d \zeta(x) \quad \text { for } \quad|\mu|-a . e \quad y \in \mathcal{G} .
$$

Then $\zeta \circ f$ is again a generalised function such that

$$
\langle\zeta \circ f, \mu\rangle=\langle f, \zeta * \mu\rangle \quad(\mu \in M(\mathcal{G}))
$$

see pages 88 and 89 of [15]. So, $\zeta \circ f$ is the generalised function corresponding to the functional $f \zeta \in M(\mathcal{G})^{*}$ such that $\Psi(\zeta \circ f)=f \zeta$. Also, by using the right convolution notation, we can show that $f \circ \zeta$ is the generalised function corresponding to the functional $\zeta f \in M(\mathcal{G})^{*}$. In what follows, we do not distinguish between the linear functionals $f \zeta$ and $\zeta f$ and their corresponding generalised functions.

Later we will need the following remark in our present investigation. 
Remark 2.2 Suppose that $B M(\mathcal{G})$ denote the Banach space of all bounded Borel measurable functions on $\mathcal{G}$ with the supremum norm $\|\cdot\|_{u}$. Then each $f \in B M(\mathcal{G})$ may be regarded as an element $\left(f_{\mu}\right)_{\mu \in M(\mathcal{G})}$ in $G L(\mathcal{G})$, where for each $\mu \in M(\mathcal{G})$ the functions $f_{\mu}$ denotes the equivalent class of $f$ in $L^{\infty}(|\mu|)$. Hence $B M(\mathcal{G})$ can be considered as a closed subspace of $G L(\mathcal{G})$ containing the space $C_{b}(\mathcal{G})$ of all complex-valued continuous bounded functions on $\mathcal{G}$. Moreover, each $f \in B M(\mathcal{G})$ may be regarded as an element in $M(\mathcal{G})^{*}$ by the pairing $\langle f, \mu\rangle=\int_{\mathcal{G}} f d \mu$ $(\mu \in M(\mathcal{G}))$. In this case, the restriction of the map $\Psi$ to $B M(\mathcal{G})$ is precisely the embedding of $B M(\mathcal{G})$ into $M(\mathcal{G})^{*}$.

\section{Generalised functions that vanish at infinity}

We commence this section by recalling the main object of the work which is introduced and studied by the authors in [10].

Definition 3.1 A generalised function $f=\left(f_{\mu}\right)_{\mu \in M(\mathcal{G})}$ vanishes at infinity if for each $\varepsilon>0$, there is a compact subset $K_{\varepsilon}$ of $\mathcal{G}$ for which $\left\|f_{\mu} \chi_{\mathcal{G} \backslash K_{\varepsilon}}\right\|_{\mu, \infty}<\varepsilon$ for all $\mu \in M(\mathcal{G})$; formally

$$
\forall \varepsilon>0 \quad \exists K_{\varepsilon} \in \mathcal{K}(\mathcal{G}) \quad \text { s.t. } \quad \forall \mu \in M(\mathcal{G}), \quad\left|f_{\mu}(x)\right|<\varepsilon \quad \text { for }|\mu|-\text { almost all } x \in \mathcal{G} \backslash K_{\varepsilon},
$$

where $\chi_{K_{\varepsilon}}$ denotes the characteristic function of $K_{\varepsilon}$ on $\mathcal{G}$ and $\mathcal{K}(\mathcal{G})$ denotes the set of all compact subsets in $\mathcal{G}$.

We denote by $G L_{0}(\mathcal{G})$ the $\mathrm{C}^{*}$-subalgebra of $G L(\mathcal{G})$ consisting of all generalised functions that vanish at infinity.

The aim of the present section is to study some aspects of $G L_{0}(\mathcal{G})$ as a $\mathrm{C}^{*}$-subalgebra of $G L(\mathcal{G})$. Let us give a simple but important result whose proof involves nothing more than routine calculations.

Lemma 3.2 Suppose that $\mathcal{K}(\mathcal{G})$ is directed downwards and for each $\alpha, u_{K_{\alpha}} \in C_{c}(\mathcal{G})$ is chosen such that $0 \leq u_{K_{\alpha}} \leq 1$ and $u_{K_{\alpha}}(x)=1$ for all $x \in K_{\alpha}$. Then $\left(u_{K_{\alpha}}\right)$ is a bounded approximate identity for $G L_{0}(\mathcal{G})$.

Our next result shows that the subspaces

$$
M(\mathcal{G}) \circ G L_{0}(\mathcal{G}):=\left\{\zeta \circ f: \zeta \in M(\mathcal{G}) \text { and } f \in G L_{0}(\mathcal{G})\right\}
$$

and

$$
G L_{0}(\mathcal{G}) \circ M(\mathcal{G}):=\left\{f \circ \zeta: \zeta \in M(\mathcal{G}) \text { and } f \in G L_{0}(\mathcal{G})\right\}
$$

of $G L(\mathcal{G})$ coincide with $G L_{0}(\mathcal{G})$.

Lemma 3.3 The following assertions hold.

(i) $M(\mathcal{G}) \circ G L_{0}(\mathcal{G})=G L_{0}(\mathcal{G})$,

(ii) $G L_{0}(\mathcal{G}) \circ M(\mathcal{G})=G L_{0}(\mathcal{G})$. 
Proof. We prove the first; the proof of the second is similar. Since the inclusion $G L_{0}(\mathcal{G}) \subseteq$ $\delta_{e} \circ G L_{0}(\mathcal{G}) \subseteq M(\mathcal{G}) \circ G L_{0}(\mathcal{G})$, it will be enough to prove the reverse inclusion. To this end, let $\zeta \in M(\mathcal{G}), f=\left(f_{\mu}\right)_{\mu \in M(\mathcal{G})} \in G L_{0}(\mathcal{G})$ and $\epsilon>0$ be given. Without loss of generality, we may assume that $\zeta$ is non-zero and positive and that $f \neq 0$. By the regularity of $\zeta$, we can choose a compact subset $K_{1}$ of $\mathcal{G}$ such that $0<\zeta\left(\mathcal{G} \backslash K_{1}\right)<\epsilon / 2\|f\|_{\infty}$. Also, since $f$ vanishes at infinity, there is a compact subset $K_{2}$ in $\mathcal{G}$ with $\left\|f-\chi_{K_{2}} f\right\|_{\infty}<\epsilon / 2\|\zeta\|$. Therefore

$$
\left\|\zeta \circ f-\left(\chi_{K_{1}} \zeta\right) \circ\left(\chi_{K_{2}} f\right)\right\|_{\infty} \leq\left\|\zeta-\chi_{K_{1}} \zeta\right\|\|f\|_{\infty}+\left\|\chi_{K_{1}} \zeta\right\|\left\|f-\chi_{K_{2}} f\right\|_{\infty} \leq \epsilon,
$$

where $\chi_{K_{1}} \zeta$ is the measure in $M(\mathcal{G})$ defined on each Borel subsets $A$ of $\mathcal{G}$ by $\chi_{K_{1}} \zeta(A)=$ $\int_{A} \chi_{K_{1}} d \zeta$. Now suppose that $\mu$ is an arbitrary element of $M(\mathcal{G})$. Observe that

$$
\left(\left(\chi_{K_{1}} \zeta\right) \circ\left(\chi_{K_{2}} f\right)\right)_{\mu}(x)=\int_{K_{1}} \chi_{K_{2}}(y x) f_{\left(\chi_{K_{1}} \zeta\right) * \mu}(y x) d \zeta(y)
$$

For each $x \in \mathcal{G} \backslash K_{1}{ }^{-1} K_{2}$, we get $K_{1} x \subseteq \mathcal{G} \backslash K_{2}$, and hence $\left(\left(\chi_{K_{1}} \zeta\right) \circ\left(\chi_{K_{2}} f\right)\right)(x)=0$ for $\mu$-almost all $x \in \mathcal{G} \backslash K_{1}{ }^{-1} K_{2}$. Thus, inequality (1) implies that

$$
\left|(\zeta \circ f)_{\mu}(x)\right|<\varepsilon, \quad \text { for } \mu-\text { almost all } x \in \mathcal{G} \backslash K_{1}{ }^{-1} K_{2} .
$$

It follows that $\zeta \circ f \in G L_{0}(\mathcal{G})$. We have now completed the proof of the lemma.

Now, let $M_{a}(\mathcal{G})$ be the closed ideal of $M(\mathcal{G})$ consisting of all absolutely continuous measures with respect to $\lambda$ and let $L^{1}(\mathcal{G})$ denote the group algebra of $\mathcal{G}$ as defined in [9, Theorem 14.17 and 14.18]. Then, the Radon-Nikodym Theorem can be interpreted as an identification of $M_{a}(\mathcal{G})$ with $\left\{\nu_{\varphi}: \varphi \in L^{1}(\mathcal{G})\right\}$, where $\nu_{\varphi}$ is the measure in $M(\mathcal{G})$ defined on each Borel subset $A$ of $\mathcal{G}$ by $\nu_{\varphi}(A)=\int_{A} \varphi d \lambda$. This allows us to show that $M_{a}(\mathcal{G})^{*}$, the first dual space of $M_{a}(\mathcal{G})$, is $L^{\infty}(\mathcal{G})$, where $L^{\infty}(\mathcal{G})$ denotes the Lebesgue space as defined in [9, Definition 12.11] equipped with the essential supremum norm. Given any $\sigma \in M_{a}(\mathcal{G})$ and $g \in L^{\infty}(\mathcal{G})$, define the complex-valued functions $g \star \sigma$ and $\sigma \star g$ on $\mathcal{G}$ by

$$
(g \star \sigma)(x)=\left\langle g, \delta_{x} * \sigma\right\rangle=\int_{\mathcal{G}} g(x y) d \sigma(y)
$$

and

$$
(\sigma \star g)(x)=\left\langle g, \sigma * \delta_{x}\right\rangle=\int_{\mathcal{G}} g(y x) d \sigma(y)
$$

for all $x \in \mathcal{G}$, where $\delta_{x}$ denotes the Dirac measure at $x$. Then, it is not hard to check that the functions $g \star \sigma$ and $\sigma \star g$ are in $C_{b}(\mathcal{G})$; this is because of, $M_{a}(\mathcal{G})$ can be identified with all $\nu \in M(\mathcal{G})$ such that the map $x \mapsto \delta_{x} *|\nu|$ and $x \mapsto|\nu| * \delta_{x}$ from $\mathcal{G}$ into $M(\mathcal{G})$ are norm continuous, see for example $\left[9,19.27\right.$ and 20.31 ]. In particular, if $\mathcal{P}: G L(\mathcal{G}) \rightarrow L^{\infty}(\mathcal{G})$ is the adjoint of the natural embedding from $M_{a}(\mathcal{G})$ into $M(\mathcal{G})$, then $\mathcal{P}$ is the restriction mapping and hence norm decreasing and onto.

For the formulation of the following statements we recall Remark 2.2 which allows us to consider $C_{b}(\mathcal{G})$ as a closed subspace of $G L(\mathcal{G})$ containing $C_{0}(\mathcal{G})$. 
Lemma 3.4 If $\sigma$ is an arbitrary element of $M_{a}(\mathcal{G})$, then for given $f=\left(f_{\mu}\right)_{\mu \in M(\mathcal{G})}$ in $G L(\mathcal{G})$ and all $\mu \in M(\mathcal{G})$, we have

(i) $(\sigma \circ f)_{\mu}=\sigma \star \mathcal{P}(f),|\mu|-a . e .$,

(ii) $(f \circ \sigma)_{\mu}=\mathcal{P}(f) \star \sigma,|\mu|-a . e$.

In particular, $(\sigma \circ f)_{\mu}$ and $(f \circ \sigma)_{\mu}$ are in $C_{b}(\mathcal{G})$ for all $\mu \in M(\mathcal{G})$.

Proof. We prove the assertion (i); the proof of (ii) is similar. First note that $\sigma \circ f$ is the generalised function $h=\left(h_{\mu}\right)_{\mu \in M(\mathcal{G})}$, where for each $\mu \in M(\mathcal{G})$ satisfies the following equality

$$
h_{\mu}(x)=(\sigma \circ f)_{\mu}(x)=l_{\sigma} f_{\sigma * \mu}(x)=\int_{\mathcal{G}} f_{\sigma * \mu}(y x) d \sigma(y) .
$$

On the other hand, for an arbitrary $\mu$ in $M(\mathcal{G})$ and any Borel subset $A$ of $\mathcal{G}$, since $\chi_{A} \mu \ll \mu$, we have

$$
\begin{aligned}
\int_{\mathcal{G}} \chi_{A} h_{\mu} d \mu & =\int_{\mathcal{G}} f_{\sigma * \chi_{A} \mu} d\left(\sigma * \chi_{A} \mu\right) \\
& =\left\langle\mathcal{P}(f), \sigma * \chi_{A} \mu\right\rangle \\
& =\int_{\mathcal{G}} \int_{\mathcal{G}} \mathcal{P}(f)(y x) d \sigma(y) d\left(\chi_{A} \mu\right)(x) \\
& =\int_{\mathcal{G}} \chi_{A}(\sigma \star \mathcal{P}(f)) d \mu .
\end{aligned}
$$

Hence, $(\sigma \circ f)_{\mu}=\sigma \star \mathcal{P}(f)|\mu|$-a.e. $(\mu \in M(\mathcal{G}))$. It follows that $\sigma \circ f \in C_{b}(\mathcal{G})$.

Now, in light of Lemmas 3.3 and 3.4, the following proposition is now immediate.

Proposition 3.5 The following assertions hold.

(i) $M_{a}(\mathcal{G}) \circ G L_{0}(\mathcal{G})=C_{0}(\mathcal{G})$,

(ii) $G L_{0}(\mathcal{G}) \circ M_{a}(\mathcal{G})=C_{0}(\mathcal{G})$.

Recall from [15, Page. 90] that a linear functional $\mathrm{m}$ in $G L(\mathcal{G})^{*}$ is called a mean if $\mathrm{m}(\mathbf{1})=1$ and $\mathrm{m}(f) \geq 0$ whenever $f \in G L(\mathcal{G})$ with $f \geq 0$, and it is topological left invariant if $\mathrm{m}(\zeta \circ f)=$ $\mathrm{m}(f)$ for all $f \in G L(\mathcal{G})$ and

$$
\zeta \in P(\mathcal{G})=\{\nu \in M(\mathcal{G}): \nu \geq 0 \text { and }\|\nu\|=1\} .
$$

In $[15$, Theorem 4.1], Wong proved that $G L(\mathcal{G})$ has a topological left invariant mean if and only if $M(\mathcal{G})^{*}$ has a topological left invariant mean. In particular, he showed that $\Psi^{*}$, the adjoint of $\Psi$, maps the set of all topological left invariant means on $M(\mathcal{G})^{*}$ onto that of $G L(\mathcal{G})$. Related to this result, we have the following result which asserts that in the case where $\mathcal{G}$ is non-compact, then any topological left invariant mean on $G L(\mathcal{G})$ lies in $G L_{0}(\mathcal{G})^{\perp}$, where here and in the sequel, $G L_{0}(\mathcal{G})^{\perp}$ denotes the following weak*-closed subspace of $G L(\mathcal{G})^{*}$

$$
\left\{\mathrm{m} \in G L(\mathcal{G})^{*}:\langle\mathrm{m}, f\rangle=0 \text { for all } f \in G L_{0}(\mathcal{G})\right\} .
$$

In fact, the next result shows that $G L_{0}(\mathcal{G})^{\perp}$ is far from devoid of interest. 
Proposition 3.6 If $\mathcal{G}$ is non-compact, then any topological left invariant mean on $G L(\mathcal{G})$ lies in $G L_{0}(\mathcal{G})^{\perp}$.

Proof. Suppose that $\mathrm{m}$ is a topological left invariant mean on $G L(\mathcal{G})$. First note that the noncompactness of $\mathcal{G}$ implies that there exists a sequence $\left(x_{n}\right)$ of disjoint elements of $\mathcal{G}$ and a compact symmetric neighborhood $V$ of $e$ such that the sets $x_{n} V$ for all $n \in \mathbb{N}$ are pairwise disjoint; see $[9,11.43(\mathrm{e})]$. Now, it is not hard to check that $\chi_{x V}=\delta_{x^{-1}} \circ \chi_{V},|\mu|-$ a.e. for all $x \in \mathcal{G}$ and $\mu \in M(\mathcal{G})$. Moreover, by Remark 2.2, for each $p \in \mathbb{N}$ the function $\sum_{n=1}^{p} \chi_{x_{n} V}=\sum_{n=1}^{p} \delta_{x_{n}-1} \circ \chi_{V}$ is in $G L(\mathcal{G})$ for which $\sum_{n=1}^{p} \chi_{x_{n} V} \leq \mathbf{1}$. It follows that

$$
p\left\langle\mathrm{~m}, \chi_{V}\right\rangle=\left\langle\mathrm{m}, \sum_{n=1}^{p} \chi_{x_{n} V}\right\rangle \leq 1 \quad(p \in \mathbb{N}) .
$$

Thus $\left\langle\mathrm{m}, \chi_{V}\right\rangle=0$ and thus we have

$$
\left\langle\mathrm{m}, \chi_{x V}\right\rangle=\left\langle\mathrm{m}, \delta_{x^{-1}} \circ \chi_{V}\right\rangle=0 \quad(x \in \mathcal{G}) .
$$

Now suppose that $f=\left(f_{\mu}\right)_{\mu \in M(\mathcal{G})}$ is a non-zero element of $G L_{0}(\mathcal{G})$. The proof will be completed by showing that $\langle\mathrm{m}, f\rangle=0$. To this end, without loss of generality, we may assume that $\|f\|_{\infty}=1$. Then, since $f^{*} f=\left(\left|f_{\mu}\right|^{2}\right)_{\mu \in M(\mathcal{G})}$ vanishes at infinity, for given $\varepsilon>0$ one can choose $y_{1}, \ldots, y_{q} \in \mathcal{G}$ such that

$$
\left|f_{\mu}\right|^{2} \leq \sum_{i=1}^{q} \chi_{y_{i} V}+\varepsilon \quad|\mu|-\text { a.e. } \quad(\mu \in M(\mathcal{G})) .
$$

Now, by considering $h=\sum_{i=1}^{q} \chi_{y_{i} V}+\varepsilon \in B M(\mathcal{G})$ as an element of $G L(\mathcal{G})$, we have $f^{*} f \leq h$, see Remark 2.1. Hence, in light of [13, Theorem 3.3.2] and equality (2), we see that

$$
|\langle\mathrm{m}, f\rangle|^{2} \leq\left\langle\mathrm{m}, f^{*} f\right\rangle \leq\langle\mathrm{m}, h\rangle \leq \varepsilon
$$

It follows that $\langle\mathrm{m}, f\rangle=0$. Hence, $\mathrm{m} \in G L_{0}(\mathcal{G})^{\perp}$.

\section{$4 G L_{0}(\mathcal{G})^{*}$ as a subalgebra of $M(\mathcal{G})^{* *}$}

As we know, there exists two natural products on $M(\mathcal{G})^{* *}$ extending the one on $M(\mathcal{G})$, known as the first and second Arens products of $M(\mathcal{G})^{* *}$. The first Arens product on $M(\mathcal{G})^{* *}$ is defined in three steps as follows. For $\mathrm{m}, \mathrm{n}$ in $M(\mathcal{G})^{* *}$, the element $\mathrm{m} \odot \mathrm{n}$ of $M(\mathcal{G})^{* *}$ is defined by

$$
\langle\mathrm{m} \odot \mathrm{n}, f\rangle=\langle\mathrm{m}, \mathrm{n} f\rangle \quad(f \in G L(\mathcal{G})),
$$

where $\langle\mathrm{m} f, \zeta\rangle=\langle\mathrm{m}, f \zeta\rangle$ and $f \zeta=\zeta \circ f$ for all $\zeta \in M(\mathcal{G})$. Equipped with this product, $M(\mathcal{G})^{* *}$ is a Banach algebra which contains $M(\mathcal{G})$ as a subalgebra. Moreover, by the duality relation between $M(\mathcal{G})$ and $G L(\mathcal{G})$, there exists a unique generalised function $h \in G L(\mathcal{G})$ such that $\mathrm{m} f=\Psi(h)$; In what follows, we denote the generalised function $\Psi^{-1}(\mathrm{~m} f)$ corresponding to $\mathrm{m} f \in M(\mathcal{G})^{*}$, by 
$\mathrm{m} f$. Moreover, $M(\mathcal{G})$ and $G L_{0}(\mathcal{G})$ are in duality with respect to the natural bilinear map given for each $\eta \in M(\mathcal{G})$ and $f=\left(f_{\mu}\right)_{\mu \in M(\mathcal{G})}$ in $G L_{0}(\mathcal{G})$ by $\langle f, \eta\rangle=\int_{\mathcal{G}} f_{\eta} d \eta$. Therefore, $M(\mathcal{G})$ may be identified with a closed subspace of $G L_{0}(\mathcal{G})^{*}$. Furthermore, if $f=\left(f_{\mu}\right)_{\mu \in M(\mathcal{G})} \in G L_{0}(\mathcal{G})$ and $\zeta \in M(\mathcal{G})$, then, by Lemma 3.3, $\zeta \circ f$ and $f \circ \zeta$ are also in $G L_{0}(\mathcal{G})$ and

$$
\langle\zeta \circ f, \nu\rangle=\langle f, \zeta * \nu\rangle, \quad \text { and } \quad\langle f \circ \zeta, \nu\rangle=\langle f, \nu * \zeta\rangle,
$$

for all $\nu \in M(\mathcal{G})$. Hence the product " $\odot$ " is well defined on $G L_{0}(\mathcal{G})^{*}$ and $G L_{0}(\mathcal{G})^{*}$ is a Banach algebra with this product, if we show that $G L_{0}(\mathcal{G})$ is a topologically introverted subspace of $G L(\mathcal{G})$. To this end, we have the following result.

Proposition 4.1 The space $G L_{0}(\mathcal{G})$ is left (right) topologically introverted in $G L(\mathcal{G})$; that is, $\mathrm{m} f \in G L_{0}(\mathcal{G})\left(f \mathrm{~m} \in G L_{0}(\mathcal{G})\right)$ for all $\mathrm{m} \in G L_{0}(\mathcal{G})^{*}$ and $f \in G L_{0}(\mathcal{G})$.

Proof. We only show that $G L_{0}(\mathcal{G})$ is a left topologically introverted subspace of $G L(\mathcal{G})$; the proof of the other assertion is similar. To this end, let $\mathrm{m} \in G L_{0}(\mathcal{G})^{*}, f=\left(f_{\mu}\right)_{\mu \in M(\mathcal{G})} \in G L_{0}(\mathcal{G})$ and $\varepsilon>0$ be given. Since $G L_{0}(\mathcal{G})$ is spanned by its positive elements, we can suppose that $\mathrm{m} \geq 0$. Also, since $f$ vanish at infinity, there is a compact set $B$ in $\mathcal{G}$ with $\left|f_{\mu}(x)\right|<\varepsilon$ for $\mu$-almost all $x \in \mathcal{G} \backslash B(\mu \in M(\mathcal{G}))$.

Now let $\varrho$ denote the restriction of $\mathrm{m}$ to $C_{0}(\mathcal{G})$. Then there exists a compact subset $K$ of $\mathcal{G}$ such that $\varrho(\mathcal{G} \backslash K)<\varepsilon / 2$. In particular, if $\mathrm{m}_{K}$ denote the continuous linear functional on $G L_{0}(\mathcal{G})$ defined by

$$
\left\langle\mathrm{m}_{K}, h\right\rangle:=\left\langle\mathrm{m}, h-u_{K} h\right\rangle \quad\left(h \in G L_{0}(\mathcal{G})\right),
$$

where $u_{K}$ is a fixed function in $C_{c}(\mathcal{G})$ such that $0 \leq u_{K} \leq 1$, and $u_{K}(x)=1$ for all $x \in K$. Then the positivity of the linear functional $\mathrm{m}_{K}$ on $G L_{0}(\mathcal{G})$ implies that $\left\|\mathrm{m}_{K}\right\|=\lim _{\alpha}\left\langle\mathrm{m}_{K}, u_{K_{\alpha}}\right\rangle$, where $\left(u_{K_{\alpha}}\right)$ is the net introduced in Lemma 3.2. Hence, there exists $\alpha_{0}$ such that

$$
\left\|\mathrm{m}_{K}\right\|-\frac{\varepsilon}{2} \leq\left\langle\mathrm{m}_{K}, u_{K_{\alpha_{0}}}\right\rangle \leq\left\|\left.\mathrm{m}_{K}\right|_{C_{0}(\mathcal{G})}\right\| .
$$

It follows that $\left\|\mathrm{m}_{K}\right\|<\varepsilon$. Indeed,

$$
\begin{aligned}
\left\|\left.\mathrm{m}_{K}\right|_{C_{0}(\mathcal{G})}\right\| & =\sup \left\{\left|\left\langle\varrho, g-u_{K} g\right\rangle\right|: g \in C_{0}(\mathcal{G}) \text { and }\|g\| \leq 1\right\} \\
& =\sup \left\{\left|\left\langle\chi_{\mathcal{G} \backslash K} \varrho,\left(g-u_{K} g\right)\right\rangle\right|: g \in C_{0}(\mathcal{G}) \text { and }\|g\| \leq 1\right\} \\
& \leq\left\|\chi_{\mathcal{G} \backslash K} \varrho\right\| \\
& =\varrho(\mathcal{G} \backslash K) .
\end{aligned}
$$

If now, $\nu$ is an arbitrary probability measure in $M(\mathcal{G})$, then $\zeta:=\left(\chi_{\mathcal{G} \backslash B K^{-1}}\right) \nu$ is a measure in $M(\mathcal{G})$ for which $\operatorname{supp}(\zeta) \subseteq \mathcal{G} \backslash B K^{-1}$. Further, choose a compact subset $D$ in $\mathcal{G}$ for which $D \subseteq \mathcal{G} \backslash B K^{-1}$ and that $|\zeta|(\mathcal{G} \backslash D)<\varepsilon$. Trivially, for each $x \in \mathcal{G} \backslash D^{-1} B$, we see that $D x \subseteq \mathcal{G} \backslash B$, and therefore for each $\mu \in M(\mathcal{G})$ we have

$$
\left|(\zeta \circ f)_{\mu}(x)\right| \leq \int_{\mathcal{G} \backslash D}\left|f_{\zeta * \mu}(y x)\right| d|\zeta|(y)+\int_{D}\left|f_{\zeta * \mu}(y x)\right| d|\zeta|(y) \leq \varepsilon\left(\|f\|_{\infty}+1\right) ;
$$


that is, $\left|(\zeta \circ f)_{\mu}(x)\right| \leq \varepsilon\left(\|f\|_{\infty}+1\right)$ for $\mu$-almost all $x \in \mathcal{G} \backslash D^{-1} B$. In particular, since $D^{-1} B \cap K=\emptyset$, we see that

$$
\left\|(\zeta \circ f) \chi_{K}\right\|_{\infty}=\sup _{\mu \in M(\mathcal{G})}\left\|(\zeta \circ f)_{\mu} \chi_{K}\right\|_{\mu, \infty} \leq \varepsilon\left(\|f\|_{\infty}+1\right)
$$

Thus

$$
\begin{aligned}
\int_{\mathcal{G} \backslash B K^{-1}}(\mathrm{~m} f)_{\zeta}(x) d \zeta(x) & =\langle\mathrm{m} f, \zeta\rangle \\
& =\left\langle\mathrm{m}, u_{K}(\zeta \circ f)\right\rangle+\left\langle\mathrm{m}_{K}, \zeta \circ f\right\rangle \\
& \leq \varepsilon\left(\|f\|_{\infty}+1\right)\|\mathrm{m}\|+\varepsilon\|\zeta\|\|f\|_{\infty}
\end{aligned}
$$

On the other hand, since $\zeta \ll \nu$, we have

$$
\int_{\mathcal{G} \backslash B K^{-1}}(\mathrm{~m} f)_{\zeta}(x) d \zeta(x)=\int_{\mathcal{G} \backslash B K^{-1}}(\mathrm{~m} f)_{\nu}(x) d \zeta(x)=\int_{\mathcal{G} \backslash B K^{-1}}(\mathrm{~m} f)_{\nu}(x) d \nu(x) .
$$

This shows that, if $\nu \in M(\mathcal{G})$, then $(\mathrm{m} f)_{\nu}(x) \leq \varepsilon\left[\left(\|f\|_{\infty}+1\right)\|\mathrm{m}\|+\|f\|_{\infty}\right]$ for $\nu$-almost all $x \in \mathcal{G} \backslash B K^{-1}$, and thus $\mathrm{m} f \in G L_{0}(\mathcal{G})$.

A linear functional $\mathrm{m}$ in $G L_{0}(\mathcal{G})^{*}$ (resp. $M(\mathcal{G})^{* *}$ ) has compact carrier if there exists a compact set $K$ in $\mathcal{G}$ such that $\langle\mathrm{m}, f\rangle=\left\langle\mathrm{m}, \chi_{K} f\right\rangle$ for all $f \in G L_{0}(\mathcal{G})$ (resp. $G L(\mathcal{G})$ ); such a compact set $K$ is called a compact carrier for $\mathrm{m}$. In the sequel, the notation $M_{c}(\mathcal{G})^{* *}$ is used to denote the norm closure of functionals in $M(\mathcal{G})^{* *}$ with compact carrier.

Now, with an argument similar to the proof of Propositions 2.6, 2.7 and Theorems 2.8 and 2.11 in [11], one can prove the following result which in particular shows that the restriction map is an isometric algebra isomorphism from $M_{c}(\mathcal{G})^{* *}$ onto $G L_{0}(\mathcal{G})^{*}$. In other word, this result allows us to view $G L_{0}(\mathcal{G})^{*}$ as a subalgebra of $M(\mathcal{G})^{* *}$.

Theorem 4.2 The following assertions hold.

(i) Functionals in $G L_{0}(\mathcal{G})^{*}$ with compact carriers are norm dense in $G L_{0}(\mathcal{G})^{*}$.

(ii) If $m$ and $n$ are elements in $G L_{0}(\mathcal{G})^{*}\left(\right.$ resp. $\left.M_{c}(\mathcal{G})^{* *}\right)$ with compact carriers $K$ and $K^{\prime}$ respectively, then $m \odot n$ has compact carrier $K K^{\prime}$.

(iii) The restriction map is an isometry and an algebra isomorphism from $M_{c}(\mathcal{G})^{* *}$ onto $G L_{0}(\mathcal{G})^{*}$.

(iv) $M(\mathcal{G})^{* *}=G L_{0}(\mathcal{G})^{*} \oplus G L_{0}(\mathcal{G})^{\perp}$. In fact, any $\mathrm{m} \in M(\mathcal{G})^{* *}$ has a unique decomposition $\mathrm{m}=\mathrm{m}_{*}+\mathrm{m}_{\perp}$, where $\mathrm{m}_{*} \in G L_{0}(\mathcal{G})^{*}, \mathrm{~m}_{\perp} \in G L_{0}(\mathcal{G})^{\perp}$ and $\|\mathrm{m}\|=\left\|\mathrm{m}_{*}\right\|+\left\|\mathrm{m}_{\perp}\right\|$. Moreover, $\mathrm{m} \geq 0$ if and only if $\mathrm{m}_{*} \geq 0$ and $\mathrm{m}_{\perp} \geq 0$.

(v) $G L_{0}(\mathcal{G})^{\perp}$ is a weak*-closed ideal of $M(\mathcal{G})^{* *}$.

(vi) $G L_{0}(\mathcal{G})^{*}$ is a left or right ideal of $M(\mathcal{G})^{* *}$ if and only if $\mathcal{G}$ is compact. 
(vii) $M(\mathcal{G})$ is a left or right ideal of $G L_{0}(\mathcal{G})^{*}$ if and only if $\mathcal{G}$ is discrete.

(viii) $M_{a}(\mathcal{G})$ is a two-sided ideal in $G L_{0}(\mathcal{G})^{*}$.

Proof. The details are omitted and we only give the proof for (ii) and (viii).

(ii) Suppose that $\mathrm{m}$ and $\mathrm{n}$ are elements in $G L_{0}(\mathcal{G})^{*}\left(\operatorname{resp} . M_{c}(\mathcal{G})^{* *}\right)$ with compact carriers $K$ and $K^{\prime}$, respectively, and that $f$ is an arbitrary element in $G L_{0}(\mathcal{G})$ (resp. $G L(\mathcal{G})$ ). First, observe that

$$
\langle\mathrm{m} \odot \mathrm{n}, f\rangle=\langle\mathrm{m}, \mathrm{n} f\rangle=\left\langle\mathrm{m}, \chi_{K}(\mathrm{n} f)\right\rangle .
$$

On the one hand, for $\mu \in M(\mathcal{G})$ we have $\zeta:=\chi_{K} \mu \ll \mu$, and so

$$
\left\langle\chi_{K}(\mathrm{n} f), \mu\right\rangle=\int_{\mathcal{G}} \chi_{K}(\mathrm{n} f)_{\mu} d \mu=\int_{\mathcal{G}}(\mathrm{n} f)_{\zeta} d \zeta=\left\langle\mathrm{n}, \chi_{K^{\prime}}(\zeta \circ f)\right\rangle .
$$

On the other hand, $\chi_{K^{\prime}}(\zeta \circ f)=\zeta \circ\left(\chi_{K K^{\prime}} f\right)$. Indeed, for each $\nu \in M(\mathcal{G})$ and $h \in C_{0}(\mathcal{G})$, we have

$$
\begin{aligned}
\left(\chi_{K K^{\prime}}(\zeta * \nu)\right)(h) & =\int_{\mathcal{G}} \chi_{K K^{\prime}} h d(\zeta * \nu) \\
& =\int_{\mathcal{G}} \int_{\mathcal{G}} \chi_{K K^{\prime}}(x y) \chi_{K}(x) h(x y) d \mu(x) d \nu(y) \\
& =\int_{\mathcal{G}} \int_{\mathcal{G}} \chi_{K^{\prime}}(y) \chi_{K}(x) h(x y) d \mu(x) d \nu(y) \\
& =\left(\zeta *\left(\chi_{K^{\prime}} \nu\right)\right)(h)
\end{aligned}
$$

and this implies that

$$
\left\langle\chi_{K^{\prime}}(\zeta \circ f), \nu\right\rangle=\left\langle\zeta \circ f, \chi_{K^{\prime}} \nu\right\rangle=\left\langle f, \chi_{K K^{\prime}}(\zeta * \nu)\right\rangle=\left\langle\zeta \circ\left(\chi_{K K^{\prime}} f\right), \nu\right\rangle .
$$

Hence by using these equalities, we have

$$
\left\langle\chi_{K}(\mathrm{n} f), \mu\right\rangle=\left\langle\mathrm{n}, \zeta \circ\left(\chi_{K K^{\prime}} f\right)\right\rangle=\left\langle\mathrm{n}\left(\chi_{K K^{\prime}} f\right), \zeta\right\rangle=\left\langle\chi_{K}\left(\mathrm{n}\left(\chi_{K K^{\prime}} f\right)\right), \mu\right\rangle .
$$

Consequently

$$
\langle\mathrm{m} \odot \mathrm{n}, f\rangle=\left\langle\mathrm{m}, \chi_{K}(\mathrm{n} f)\right\rangle=\left\langle\mathrm{m}, \chi_{K}\left(\mathrm{n}\left(\chi_{K K^{\prime}} f\right)\right)\right\rangle=\left\langle\mathrm{m}, \mathrm{n}\left(\chi_{K K^{\prime}} f\right)\right\rangle=\left\langle\mathrm{m} \odot \mathrm{n}, \chi_{K K^{\prime}} f\right\rangle .
$$

It follows that $\mathrm{m} \odot \mathrm{n}$ has compact carrier $K K^{\prime}$.

(viii) That $M_{a}(\mathcal{G})$ is a closed subalgebra of $G L_{0}(\mathcal{G})^{*}$ is trivial. Now, suppose that $\sigma \in M_{a}(\mathcal{G})$ and $\mathrm{m} \in G L_{0}(\mathcal{G})^{*}$. We show that $\mathrm{m} \odot \sigma \in G L_{0}(\mathcal{G})^{*} ;$ that $\sigma \odot \mathrm{m} \in G L_{0}(\mathcal{G})^{*}$ is similar. Let $\zeta$ denote the restriction of $\mathrm{m}$ to $C_{0}(\mathcal{G})$. Since $M_{a}(\mathcal{G})$ is an ideal in $M(\mathcal{G})$, we have $\zeta * \sigma \in M_{a}(\mathcal{G})$. We now invoke Proposition 3.5 to conclude that

$$
\langle\mathrm{m} \odot \sigma, f\rangle=\langle\zeta, f \circ \sigma\rangle=\langle\zeta * \sigma, f\rangle \quad\left(f \in G L_{0}(\mathcal{G})\right),
$$

whence $\mathrm{m} \odot \sigma=\zeta * \sigma \in M_{a}(\mathcal{G})$. 
As usual, for a locally compact space $X$, we say that a subset $S \subseteq M(X)$, the Banach space of all complex regular Borel measures on $X$, is solid with respect to absolute continuity, if $t \in S$ wherever $t \ll s$, for some $s \in S$. Now, as an application of Theorem 4.2 above, by a method similar to that of [8, Lemma 5 and Theorem 6$]$, one can obtain the following generalization of that theorem; The reader will remark that the compactness of $\mathcal{G}$ is assumed in that proof only to conclude that $M_{a}(\mathcal{G})$ is an ideal in $M(\mathcal{G})^{* *}$ whereas $M_{a}(\mathcal{G})$ always is an ideal of $G L_{0}(\mathcal{G})^{*}$. The details are omitted.

Theorem 4.3 $M_{a}(\mathcal{G})$ is the unique minimal proper closed subset of $G L_{0}(\mathcal{G})^{*}$ which is an algebraic ideal and a solid set with respect to absolute continuity in $G L_{0}(\mathcal{G})^{*}$.

Next we turn our attention to the study of left (weakly) completely continuous elements of $G L_{0}(\mathcal{G})^{*}$. To this end, recall that if $\mathcal{A}$ is a Banach algebra, then $a \in \mathcal{A}$ is said to be a left (weakly) completely continuous element of $\mathcal{A}$ whenever the operator $\ell_{a}: b \mapsto a b$ is (weakly) compact operator on $\mathcal{A}$.

In what follows, for $I \subseteq G L_{0}(\mathcal{G})^{*}$, the left annihilator of $I$ is denoted by $\operatorname{lan}(I)$ and defined by

$$
\operatorname{lan}(I)=\left\{\mathrm{I} \in G L_{0}(\mathcal{G})^{*}: \mathrm{I} \odot I=\{0\}\right\},
$$

also, the right annihilator of $I$ is denoted by $\operatorname{ran}(I)$ and define by

$$
\operatorname{ran}(I)=\left\{r \in G L_{0}(\mathcal{G})^{*}: I \odot r=\{0\}\right\} .
$$

Moreover, the letter $L_{0}^{\infty}(\mathcal{G})$ means that the $\mathrm{C}^{*}$-subalgebra of $L^{\infty}(\mathcal{G})$ consisting of all functions $g$ on $\mathcal{G}$ such that for each $\varepsilon>0$, there is a compact subset $K$ of $\mathcal{G}$ for which $|g(x)|<\varepsilon$ for all $x \in \mathcal{G} \backslash K$.

Theorem 4.4 The following assertions hold.

(i) If $\sigma \in M_{a}(\mathcal{G})$, then $\sigma$ is a left (weakly) completely continuous element of $M_{a}(\mathcal{G})$ if and only if $\sigma$ is a left (weakly) completely continuous element of $G L_{0}(\mathcal{G})^{*}$.

(ii) Any left (weakly) completely continuous element $\mathrm{m}$ of $G L_{0}(\mathcal{G})^{*}$ has the form $\mathrm{m}=\sigma+\mathrm{r}$ for some $\sigma \in M_{a}(\mathcal{G})$ and $\mathrm{r} \in \operatorname{ran}\left(\mathcal{P}^{*}\left(L_{0}^{\infty}(\mathcal{G})^{*}\right)\right)$.

Proof. We only give the proof for left completely continuous element.

(i) The direct implication being trivial, we give the proof of the backward implication only. To this end, suppose that $\sigma \in M_{a}(\mathcal{G})$ is a left completely continuous element of $M_{a}(\mathcal{G})$. Then, the closure of the following set is compact in $M_{a}(\mathcal{G})$

$$
\left\{\sigma * v: v \in M_{a}(\mathcal{G}),\|v\| \leq 1\right\} \text {. }
$$

On the other hand, if $\left(e_{\alpha}\right)_{\alpha}$ is an approximate identity for $M_{a}(\mathcal{G})$ bounded by one, then for each $\alpha$ and $\mathrm{m} \in G L_{0}(\mathcal{G})^{*}$ with $\|\mathrm{m}\| \leq 1$, we have

$$
\left\|\sigma \odot \mathrm{m}-\sigma *\left(e_{\alpha} \odot \mathrm{m}\right)\right\| \leq\left\|\sigma-\sigma * e_{\alpha}\right\| .
$$


This together with the fact that $M_{a}(\mathcal{G})$ is an ideal in $G L_{0}(\mathcal{G})^{*}$ implies that

$$
\left\{\sigma \odot \mathrm{m}: \mathrm{m} \in G L_{0}(\mathcal{G})^{*},\|\mathrm{~m}\| \leq 1\right\} \subseteq \overline{\left\{\sigma * v: v \in M_{a}(\mathcal{G}),\|v\| \leq 1\right\}^{M_{a}(\mathcal{G})}} .
$$

Thus the operator $\ell_{\sigma}: G L_{0}(\mathcal{G})^{*} \rightarrow G L_{0}(\mathcal{G})^{*}$ is compact.

(ii) Suppose that $\mathrm{m}$ is a left completely continuous element of $G L_{0}(\mathcal{G})^{*}$. Then, since $M_{a}(\mathcal{G})$ is an ideal in $G L_{0}(\mathcal{G})^{*}$, the operator $\left.\ell_{\mathrm{m}}\right|_{M_{a}(\mathcal{G})}$ is a compact operator on $M_{a}(\mathcal{G})$. From this, we can conclude that there exists $\sigma \in M_{a}(\mathcal{G})$ such that $\ell_{\mathrm{m}}=\ell_{\sigma}$ on $M_{a}(\mathcal{G})$, see [1]. In particular, Proposition 3.5 implies that $\langle\mathrm{m}, f\rangle=\langle\sigma, f\rangle$ for all $f \in C_{0}(\mathcal{G})$, and thus we have $v \odot \mathrm{m}=v * \sigma$ $\left(v \in M_{a}(\mathcal{G})\right)$. We now invoke the weak*-density of $M_{a}(\mathcal{G})$ in $\mathcal{P}^{*}\left(L_{0}^{\infty}(\mathcal{G})^{*}\right)$ to conclude that $\mathcal{P}^{*}\left(L_{0}^{\infty}(\mathcal{G})^{*}\right) \odot r=0$, where $r=m-\sigma$. That is $r \in \operatorname{ran}\left(\mathcal{P}^{*}\left(L_{0}^{\infty}(\mathcal{G})^{*}\right)\right)$.

In [12, Page 467], Losert by the use of the $\mathrm{C}^{*}$-algebraic structure of $M(\mathcal{G})^{*}$ proved that $M(\mathcal{G})^{* *}$ has a non-zero left (weakly) completely continuous element if and only if $\mathcal{G}$ is compact. Related to this result, we have the following result for $G L_{0}(\mathcal{G})^{*}$ where our approach in its proof is totally different from the Losert's result and relies on the theory of generalised functions.

Theorem 4.5 The following conditions are equivalent.

(i) $\mathcal{G}$ is compact.

(ii) $G L_{0}(\mathcal{G})^{*}$ has a non-zero left completely continuous element.

(iii) $G L_{0}(\mathcal{G})^{*}$ has a non-zero left weakly completely continuous element.

Proof. We need only to show that (iii) implies (i). Indeed, if $\mathcal{G}$ is compact, then $M(\mathcal{G})^{* *}=$ $G L_{0}(\mathcal{G})^{*}$ and the normalized Haar measure $\mathrm{m}$ on $\mathcal{G}$ is a left (weakly) completely continuous element of $G L_{0}(\mathcal{G})^{*}$ and (ii) $\Rightarrow$ (iii) is trivial. To this end, suppose that $\mathrm{m}$ is a nonzero left weakly completely continuous element of $G L_{0}(\mathcal{G})^{*}$. Then the set $\left\{\mathrm{m} \odot \delta_{x}: x \in \mathcal{G}\right\}$ is weakly compact and therefore $\left\{\left|\mathrm{m} \odot \delta_{x}\right|: x \in \mathcal{G}\right\}$ is weakly compact in $G L_{0}(\mathcal{G})^{*}$ by Dieudonne's characterization of weakly compact subsets; see [5, Theorem 4.22.1]. It follows that $\mathcal{E}:=\left\{|\mathrm{m}| \odot \delta_{x}: x \in \mathcal{G}\right\}$ is weakly compact; this is because of, $\left|\mathrm{m} \odot \delta_{x}\right|=|\mathrm{m}| \odot \delta_{x}$ for all $x \in \mathcal{G}$. Now we apply the KerinSmulyan theorem [2] to infer that the closed convex hull $\mathcal{K}$ of $\mathcal{E}$ is weakly compact in $G L_{0}(\mathcal{G})^{*}$. On the other hand, it is easy to see that the map $T_{x}: \mathcal{K} \rightarrow \mathcal{K}$ defined by $T_{x}(\mathrm{n})=\mathrm{n} \odot \delta_{x}$ is affine for all $x \in \mathcal{G}$. Moreover, we have

$$
\begin{aligned}
\left\|\mathrm{n} \odot \delta_{x}\right\| & =\sup \left\{\left|\left\langle\mathrm{n} \odot \delta_{x}, f\right\rangle\right|: f \in G L_{0}(\mathcal{G})^{*},\|f\|_{\infty} \leq 1\right\} \\
& =\sup \left\{\left|\left\langle\mathrm{n}, f \circ \delta_{x}\right\rangle\right|: f \in G L_{0}(\mathcal{G})^{*},\|f\|_{\infty} \leq 1\right\} \\
& =\sup \left\{|\langle\mathrm{n}, h\rangle|: h \in G L_{0}(\mathcal{G})^{*},\|h\|_{\infty} \leq 1\right\} \\
& =\|\mathrm{n}\|,
\end{aligned}
$$

for all $\mathrm{n} \in G L_{0}(\mathcal{G})^{*}$. It follows that the map $T_{x}$ is distal for all $x \in \mathcal{G}$. So there exists a fixed point $\mathrm{q} \in \mathcal{K}$ for the maps $T_{x}(x \in \mathcal{G})$; that is, $\mathrm{q} \odot \delta_{x}=\mathrm{q}$ for all $x \in \mathcal{G}$ by the Ryll-Nardzewski fixed point Theorem; see [2, Theorem 10.8]. In particular, $\mathrm{q}=\sum_{i=1}^{t} a_{i}|\mathrm{~m}| \odot \delta_{x_{i}}$ for some $x_{1}, \ldots, x_{t} \in \mathcal{G}$ 
and $a_{1}, \ldots, a_{t}$ with $\sum_{i=1}^{t} a_{i}=1$. Now, if $\left(K_{\alpha}\right)$ denotes the family of compact subsets of $\mathcal{G}$ ordered by the upward inclusion, then $\left(\chi_{K_{\alpha} x^{-1}}\right)$ is a bounded approximate identity for $G L_{0}(\mathcal{G})$ for all $x \in \mathcal{G}$. Thus

$$
\begin{aligned}
\left\|\mathrm{q} \odot \delta_{x}\right\|=\left\|\sum_{i=1}^{t} a_{i}|\mathrm{~m}| \odot \delta_{x_{i} x}\right\| & =\lim _{\alpha} \sum_{i=1}^{t} a_{i}\left\langle|\mathrm{~m}| \odot \delta_{x_{i} x}, \chi_{K_{\alpha}}\right\rangle \\
& =\sum_{i=1}^{t} a_{i} \lim _{\alpha}\left\langle|\mathrm{m}|, \chi_{K_{\alpha} x_{i} x^{-1}}\right\rangle=\|\mathrm{m}\| .
\end{aligned}
$$

Therefore $\|\mathrm{q}\|=\|\mathrm{m}\|$; since $\mathrm{m} \neq 0$, it follows that $\mathrm{q} \neq 0$.

To prove (i), suppose on the contrary that $\mathcal{G}$ is not compact and that $\overline{\mathrm{q}}$ is an extension of q from $G L_{0}(\mathcal{G})$ to a positive functional with the same norm on $G L(\mathcal{G})$; see for example [13], Theorem 3.3.8. Then, by the same manner as in the proof of Proposition 3.6, one can show that $\left.\overline{\mathrm{q}}\right|_{G L_{0}(\mathcal{G})}=0$. This implies that $\mathrm{q}=0$ a contradiction. We have now completed the proof of the theorem.

We conclude this work by the following result which is of interest in its own right. In this proposition, the notation $C_{0}(\mathcal{G})^{\perp}$ is used to denote the set of all $m \in G L_{0}(\mathcal{G})^{*}$ such that $\left.\mathrm{m}\right|_{C_{0}(\mathcal{G})}=0$ and

$$
\mathcal{E}_{1}(\mathcal{G})=\left\{E \in L_{0}^{\infty}(\mathcal{G})^{*}:\|E\|=1 \text { and } E \text { is a right identity for } L_{0}^{\infty}(\mathcal{G})^{*}\right\} .
$$

It should be noted that $E \in \mathcal{E}_{1}(\mathcal{G})$ if and only if it is a weak*-cluster point of an approximate identity in $M_{a}(\mathcal{G})$ bounded by one; see [11].

Proposition 4.6 $G L_{0}(\mathcal{G})^{*}$ is commutative if and only if $\mathcal{G}$ is discrete and abelian.

Proof. The necessity of the condition " $G L_{0}(\mathcal{G})^{*}$ is commutative" is clear. We prove its sufficiency. To this end, suppose that $G L_{0}(\mathcal{G})^{*}$ is commutative. That $\mathcal{G}$ is abelian follows trivially. In order to prove that $\mathcal{G}$ is discrete, we note that Proposition 3.5 together with the fact that the right translations on $G L_{0}(\mathcal{G})^{*}$ are weak*-continuous implies that

$$
\operatorname{ran}\left(\mathcal{P}^{*}\left(L_{0}^{\infty}(\mathcal{G})^{*}\right)\right)=C_{0}(\mathcal{G})^{\perp}
$$

Moreover, by another application of Proposition 3.5 one can obtain that $\mathcal{P}^{*}(E)-\delta_{e} \in C_{0}(\mathcal{G})^{\perp}$ for all $E \in \mathcal{E}_{1}(\mathcal{G})$. On the other hand, from the commutativity of $G L_{0}(\mathcal{G})^{*}$ we get that $C_{0}(\mathcal{G})^{\perp}=$ $\operatorname{lan}\left(\mathcal{P}^{*}\left(L_{0}^{\infty}(\mathcal{G})^{*}\right)\right)$. We therefore have

$$
\mathcal{P}^{*}(E)-\delta_{e} \in \operatorname{lan}\left(\mathcal{P}^{*}\left(L_{0}^{\infty}(\mathcal{G})^{*}\right)\right) \quad \forall E \in \mathcal{E}_{1}(\mathcal{G}) .
$$

It follows that each elements of $\mathcal{P}^{*}\left(\mathcal{E}_{1}(\mathcal{G})\right)$ is also a left identity for $\mathcal{P}^{*}\left(L_{0}^{\infty}(\mathcal{G})^{*}\right)$. We now invoke parts (ii) and (iii) of $\left[11\right.$, Theorem 2.11] to conclude that $M_{a}(\mathcal{G})=M(\mathcal{G})$. This implies that $\mathcal{G}$ is discrete. 


\section{References}

[1] C. A. Akemann, Some mapping properties of the group algebras of a compact group, Pacific J. Math. 22 (1967), 1-8.

[2] J. W. Conway, A Course in Functinal Analysis, Graduate texts in Math., Springer- Verlag, New York, 1985.

[3] H. G. Dales, A. T.-M. Lau, The second duals of Beurling algebras, Mem. Amer. Math. Soc. 177 (2005), $1-191$.

[4] H. G. Dales, A. T.-M. Lau and D. Strauss, Second duals of measure algebras, Dissertationes Math. (Rozprawy Mat.) 481 (2012), 1-121.

[5] R. E. Edwards, Functional Analysis, Holt, Rinehart and Winston, New York, 1965.

[6] G. H. Esslamzadeh, H. Javanshiri and R. Nasr-Isfahani, Locally convex algebras which determine a locally compact group, Studia Math. 233 (3), (2016), 197-207.

[7] F. Ghahramani and A. T.-M. Lau, Multipliers and ideal in second conjugate algebra related to locally compact groups, J. Funct. Anal. 132 (1995), 170-191.

[8] F. Ghahramani and J. P. McClure, The second dual algebra of the measure algebra of a compact group, Bull. London Math. Soc. 29 (1997), 223-226.

[9] E. Hewitt and K. Ross, Abstract Harmonic Analysis I, Springer, Berlin, 1970.

[10] H. Javanshiri and R. Nasr-Isfahani, The strong dual of measure algebras with certain locally convex topologies, Bull. Aust. Math. Soc. 87 (2013), 353-365.

[11] A. T.-M. Lau and J. Pym, Concerning the second dual of the group algebra of a locally compact group, $J$. London Math. Soc. 41 (1990), 445-460.

[12] V. Losert, Weakly compact multipliers on group algebras, J. Funct. Anal. 213 (2004), 466-472.

[13] G. J. Murphy, C*-Algebras and Operator Theory, Academic Press, London 1990.

[14] Yu. A. Šreider, The structure of maximal ideals in rings of measures with convolution.(Russian) Math. Sbornik (NS) 27 (69) (1950), 297-318. English translations (1953) in: Math. Soc. Transl. (First series) No. $81,365-391$.

[15] J. C. Wong, Abstract harmonic analysis of generalised functions on locally compact semigroups with applications to invariant means, J. Austral. Math. Soc. Ser. A. 23 (1977), 84-94.

[16] J. C. Wong, Convolution and separate continuity, Pacific J. Math. 75 (1978), 601-611.

HOSSEIN JAVANSHIRI

Department of Mathematics, Yazd University, P.O. Box: 89195-741, Yazd, Iran

e-mail: h.javanshiri@yazd.ac.ir

RASOUl NASR-ISFAHANI

Department of Mathematical Sciences, Isfahan University of Technology, Isfahan 84156-83111, Iran

e-mail: isfahani@cc.iut.ac.ir 